\title{
Error-Based Teaching Approach Decreases Vessel Anastomosis Errors: A Pilot Study
}

\author{
Eric de Haas, BSc, MBT ${ }^{1}$ jill P. Stone, MD, FRCSC ${ }^{2}$ William de Haas, MD, FRCSC ${ }^{2}$ \\ Christiaan H. Schrag, MD, FRCSC ${ }^{2}$
}

${ }^{1}$ Medical School Class of 2020 Program, Cumming School of Medicine, University of Calgary, Calgary, Alberta, Canada

2 Division of Plastic Surgery, Cumming School of Medicine, University of Calgary, Calgary, Alberta, Canada

Address for correspondence Eric de Haas, BSc, MBT, Medical School Class of 2020 Program, Cumming School of Medicine, University of Calgary (e-mail: ejdehaas@ucalgary.ca).

J Reconstr Microsurg Open 2019;4:e73-e76.

\begin{abstract}
Background Microsurgical anastomosis of vessels is a challenging skill that surgical residents should practice on models before attempting in the clinical setting. These skills are often taught using synthetic materials, animal tissue, or live animal models. With increasing constraints on surgical resident's time, it is important to maximize efficiency of microsurgical training. The purpose of this study is to determine if teaching surgical residents about common vessel anastomosis errors decreases the total number of suture errors during a 4-day training course.

Methods Plastic surgery residents (R1-R3) were randomly assigned to receive additional teaching focused on either common microsurgical errors or traditional microsurgical manuals. The residents then performed anastomosis on rat femoral arteries in which the total number of sutures and errors were recorded by staff microsurgeons who were blinded to the intervention.

Results Residents who received teaching on common microsurgical errors performed a total of 73 sutures of which 12 were errors. The control group who studied using traditional microsurgical manuals performed a total of 125 sutures of which 38 were errors. There was a statistically significant decrease in the total number of suture errors (Fisher's exact test; $p$-value $=0.04$ ) and in the number of partial depth bite errors (Fisher's exact test $p$-value $=0.03$ ).

\section{Keywords}

- microsurgery

- vessel anastomosis

- surgical training

Conclusion Teaching surgical residents about common vessel anastomosis errors decreased the total number of errors when compared with traditional education methods using microsurgery manuals. Partial depth bite errors were also decreased through error-based teaching.
\end{abstract}

The anastomosis of small vessels requires familiarity with procedural steps as well as precise manual dexterity. ${ }^{1}$ As such, microsurgery is a challenging skill to acquire and should be taught outside the operating room. ${ }^{2}$ Previous studies show that laboratory training of microsurgery techniques is an effective way to increase microsurgical performance and improve patency of anastomosis. ${ }^{2}$ Many models such as virtual

received

June 3, 2019

accepted after revision

September 29, 2019
DOI https://doi.org/

10.1055/s-0039-3400244. ISSN 2377-0813. reality, synthetic materials, nonliving, and living animal models are effective materials for microsurgical education., ${ }^{2,3}$ However, living rat femoral artery models are widely accepted as the gold standard for microsurgical training. ${ }^{4}$

Current obstacles for microsurgical training include procedural complexity, trainee time constraints, and high cost associated with equipment. ${ }^{3,5}$ Traditionally, resident training
Copyright $\odot 2019$ by Thieme Medical Publishers, Inc., 333 Seventh Avenue, New York, NY 10001, USA. Tel: +1(212) 584-4662.
License terms

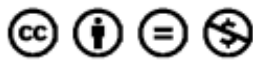


has been measured through checklists and global assessment scales; however, few manuals specifically identify the types of anastomosis errors. ${ }^{2,6}$ In a previous publication, errors commonly committed by surgical residents were identified and a visual teaching tool was created. ${ }^{7}$ Through utilizing a pictorial representation of common errors and an approach to prevent or repair them, trainees may be able to perform better in the laboratory setting as well as the clinical setting. We sought to investigate this "what-not-to-do" approach and its applicability in the microsurgery training module.

\section{Methods}

Five plastic surgery residents (R1-R3) were randomly assigned to the control or experimental group. Three were placed in the control group and two in the experimental group. Both groups were given standard microsurgery manuals to review before participating in a 4-day rat femoral artery anastomosis training program.

Before beginning the first session, the experimental group was provided with a 15-minute didactic lesson from a staff microsurgeon on common microsurgical anastomosis errors and how to avoid them. The lesson included images and explanations of errors including backwall, partial depth bite, irregular widths, tear during placement, mistaken vessel wall, and sutures that are too loose. The control group was given these 15 minutes for additional review time on standard microsurgery manuals without a staff preceptor. A total of four training sessions were offered over a 1 -week period. During each session, staff microsurgeons directly observed and cataloged all technical errors and well-placed sutures of each participant. The staff observers were blinded as to which group the residents were in and each session was overseen by a different staff physician.

Rat femoral arteries were exposed inferior to the inguinal ligament. The femoral arteries were clamped at the proximal end of the incision, and then were divided. The participants were observed by a staff microsurgeon as they performed an end-to-end anastomosis of the vessel under $10 \times$ to $20 \times$ magnification (Zeiss, Germany) using 10-0 nylon (Sharpoint, MA) and regular microsurgical instruments (Accurate Surgical \& Scientific Instruments, CA). Two simple interrupted sutures were placed at 0 and 180 degrees, and then three sutures were placed in the anterior wall. Following completion of the anterior wall, the vessel was rotated to expose the posterior wall and it was sutured with a further three sutures.

After placement of each suture, the staff microsurgeon inspected the knot both externally and internally for signs of suture errors. Real-time feedback was given to the student by first asking them to identify their error. Sutures were removed and repeated until the participant had successfully demonstrated recognition of the error and ability to correct it. When participants were satisfied with the quality of the anastomosis, the vessel clamps were released, and the rat femoral artery was inspected for locations of excess bleeding. If excess bleeding was present, the vessel was further inspected to determine the site and cause of the bleeding. Finally, the vessel was divided proximally and distally to the anastomosis for further inspection of each suture.
Each suture error was categorized as either "well placed" or subcategorized into a specific error type. These error types included vessel backwall, partial depth bite, irregular widths, tear during placement, mistaken vessel wall, too loose, or miscellaneous. The number of sutures and errors were analyzed using nonparametric statistical testing through the use of a Fisher's exact test. All animals were treated humanely and in accordance with the Canadian Council on Animal Care Ethics Approval.

\section{Results}

The control group had three participants (R1, R2, R3) who attended 4,2 , and 2 sessions, respectively, and completed a total of 125 sutures. The experimental group had two participants (R1 and R2) who attended 4 and 3 sessions, respectively, and completed a total of 73 sutures ( - Table $\mathbf{1}$ ). Of the 125 sutures the control group performed, a total of 38 were suture errors. Of the 73 sutures the experimental group performed, a total of 12 were suture errors. The average number of errors per participant session is shown in -Fig. 1.

A Fisher exact test found a significant difference between the total number of suture errors between the experimental and control groups ( $p=0.04$, - Table 2). The Fisher's exact test also showed a significant difference between the number of partial depth errors between the experimental and control groups ( $p=0.03,-$ Table 3 ). In the Fisher's exact tests, the specific error types were compared with the total number of sutures from all other categories combined. Each error type was calculated separately, and the results are summarized in -Table 4.

\section{Discussion}

Microsurgery requires a high level of technical skill and has a known increased propensity for errors and complications during the training process. ${ }^{2,8,9}$ To increase microsurgical skill, microsurgical laboratory training is often utilized and has been effective in previous studies. ${ }^{4,6,8,10}$ In addition to learning from experience, medical trainees also believe they learn from mistakes that others have made. ${ }^{11,12}$ Knowing these mistakes allows trainees to take steps to avoid committing those errors themselves. ${ }^{12,13}$ The results of our pilot study suggest that teaching residents about common microsurgical errors may improve training by reducing the occurrence of suture errors.

Table 1 Total values for control and experimental participants

\begin{tabular}{|l|l|l|}
\hline & Control & Experimental \\
\hline Surgery resident participants & 3 & 2 \\
\hline Total participant sessions & 8 & 7 \\
\hline Total sutures & 125 & 73 \\
\hline Well placed sutures & 87 & 61 \\
\hline Suture errors & 38 & 12 \\
\hline
\end{tabular}




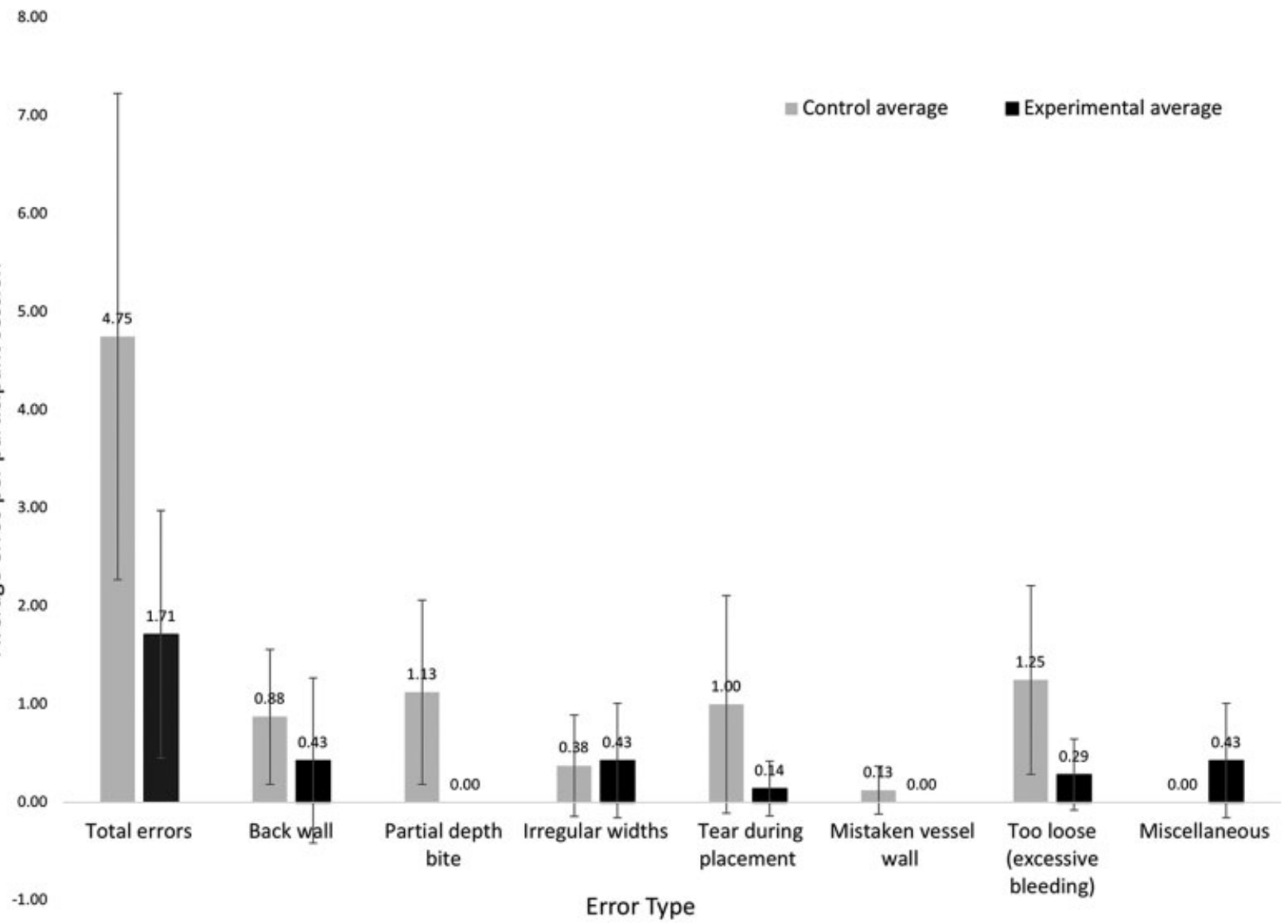

Fig. 1 Average errors of each type per participant session for the control group $(n=8)$ and the experimental group $(n=7)$. Error bars represent the standard deviation.

Table 2 Fisher exact test comparison of total errors and well-placed sutures between control and experimental groups $(p$-value $=0.04)$

\begin{tabular}{|l|l|l|}
\hline & Total errors & Well placed \\
\hline Control & 38 & 87 \\
\hline Experimental & 12 & 61 \\
\hline
\end{tabular}

Current microsurgical literature describes suturing mistakes such as backwall bites, loose sutures, and vessel wall holes. ${ }^{1,10}$ However, training manuals do not mention errors such as partial depth bites and mistaken vessel wall. In this study, the experimental group was taught how to avoid each of these suture error types and how they can be rectified. The results demonstrated that plastic surgery residents who were educated on how to avoid these errors had significantly

Table 3 Fisher's exact test comparison of partial depth errors and not partial depth errors between control and experimental groups $(p$-value $=0.03)$

\begin{tabular}{|l|l|l|}
\hline & $\begin{array}{l}\text { Partial depth } \\
\text { error }\end{array}$ & $\begin{array}{l}\text { Not partial } \\
\text { depth error }\end{array}$ \\
\hline Control & 9 & 116 \\
\hline Experimental & 0 & 73 \\
\hline
\end{tabular}

fewer total errors. They also had less partial depth errors when compared with the control group.

Partial depth errors are caused when the suture does not pass through the entire vessel wall into the lumen. ${ }^{7,14}$ This often occurs because the adventitia is confused for vessel wall and results in improper approximation of the two sides. ${ }^{7}$

Table 4 Fisher exact test comparison of total errors and wellplaced sutures between control and experimental groups

\begin{tabular}{|l|l|}
\hline & $\begin{array}{l}\text { Fisher's exact test } \\
\text { statistic value }\end{array}$ \\
\hline Total errors & $0.04^{\mathrm{a}}$ \\
\hline Back wall error & 0.75 \\
\hline Partial depth error & $0.03^{\mathrm{a}}$ \\
\hline Irregular widths & 0.67 \\
\hline Tear during placement & 0.16 \\
\hline Mistaken vessel wall & 1.00 \\
\hline Too loose (excessive bleeding) & 0.22 \\
\hline Miscellaneous error & $0.05^{\mathrm{a}}$ \\
\hline
\end{tabular}

Note: Each error type was compared between control and experimental groups using the number of errors of each type, paired with number of sutures from all other categories combined.

${ }^{a}$ Significance was determined at a $p$-value of $<0.05$. 
During training, the experimental group was informed that proper dissection of the adventitia away from the vessel end will decrease the occurrence of these partial depth errors.

This study reached statistical significance for the reduction of total errors and partial depth errors. However, the small sample size limits the power of the results and required nonparametric statistical analysis. Another limitation of this study is that there were unequal number of residents in the control and experimental group and they attended a different number of total training sessions. Strengths of this study include direct observation of residents by blinded staff microsurgeons. The staff surgeons were familiar with each error type and were able to determine the quality of sutures in real time. Furthermore, the use of a high-fidelity living rat femoral artery model allowed the patency and approximation of the anastomosis to be tested. ${ }^{15}$

Microsurgical education manuals provide step-by-step instructions on how to perform vessel anastomosis. However, they do not highlight common mistakes or the best ways to avoid or revise them. This study demonstrated that a 15-minute didactic lesson focusing on common suturing errors and how to avoid them, can decrease the total number of vessel anastomosis suture errors.

\section{Conclusion}

Residents who were randomized to receive specific training on microsurgical suturing errors had fewer total errors as well as less partial depth errors when compared with the control group. This information will help both microsurgery staff and trainees in effective microsurgical education.

\section{Conflict of Interest}

None declared.

\section{Acknowledgments}

The authors would like to thank Shivani Brown for her expert assistance in data analysis and editing. The authors would also like to thank Dr. Duncan Nickerson and Dr. Don MacPhalen for observing participants during the microsurgery course.

\section{References}

1 Chan W, Niranjan N, Ramakrishnan V. Structured assessment of microsurgery skills in the clinical setting. J Plast Reconstr Aesthet Surg 2010;63(08):1329-1334

2 Balasundaram I, Aggarwal R, Darzi LA. Development of a training curriculum for microsurgery. Br J Oral Maxillofac Surg 2010;48 (08):598-606

3 Bates BJ, Wimalawansa SM, Monson B, Rymer MC, Shapiro R, Johnson RM. A simple cost-effective method of microsurgical simulation training: the turkey wing model. J Reconstr Microsurg 2013;29(09):615-618

4 Sidhu RS, Park J, Brydges R, MacRae HM, Dubrowski A. Laboratorybased vascular anastomosis training: a randomized controlled trial evaluating the effects of bench model fidelity and level of training on skill acquisition. J Vasc Surg 2007;45(02):343-349

5 Hirschl RB. The making of a surgeon: 10,000 hours? J Pediatr Surg 2015;50(05):699-706

6 Ramachandran S, Ghanem AM, Myers SR. Assessment of microsurgery competency-where are we now? Microsurgery 2013;33 (05):406-415

7 Stone JP, Doherty CC, Schrag CH. Incidence and type of errors in microsurgical technique in surgical trainees. J Reconstr Microsurg 2016;32(07):528-532

8 Trignano E, Fallico N, Zingone G, Dessy LA, Campus GV. Microsurgical training with the three-step approach. J Reconstr Microsurg 2017;33(02):87-91

9 Ko J-W, Lorzano A, Mirarchi AJ. Effectiveness of a microvascular surgery training curriculum for orthopaedic surgery residents. J Bone Joint Surg Am 2015;97(11):950-955

10 Furka I, Brath E, Nemeth N, Miko I. Learning microsurgical suturing and knotting techniques: comparative data. Microsurgery 2006;26(01):4-7

11 Fischer MA, Mazor KM, Baril J, Alper E, DeMarco D, Pugnaire M. Learning from mistakes. Factors that influence how students and residents learn from medical errors. J Gen Intern Med 2006;21 (05):419-423

12 Gardner A, Rich M. Error management training and simulation education. Clin Teach 2014;11(07):537-540

13 Maerz L. Avoiding common surgical errors. Ann Surg 2006;244 (05):837

14 Pafitanis G, Veljanoski D, Ghanem AM, Myers S. Intimal surface suture line (end-product) assessment of end-to-side microvascular anastomosis. Plast Reconstr Surg Glob Open 2017;5(07): e1409

15 Austin GT, Hammond FW, Schaberg SJ, Scharpf HO. A laboratory model for vascular microsurgery. J Oral Maxillofac Surg 1983;41 (07):450-455 